\title{
Complexity of Polynomial Multiplication over Finite Fields
}

\author{
Michael Kaminski \\ Department of Computer Science, \\ Technion - Israel Institute of Technology, \\ Haifa 32000, Israel \\ kaminski@cs.technion.ac.il
}

\begin{abstract}
We prove the $\left(3+\frac{(q-1)^{2}}{q^{5}+(q-1)^{3}}\right) n-o(n)$ lower bound on the quadratic complexity of multiplication of two degree- $n$ polynomials over a $q$-element field. The proof is based on a novel combination of two known techniques. One technique is the analysis of Hankel matrices representing bilinear forms defined by linear combinations of the coefficients of the polynomial product. The other technique is a counting argument from the coding theory.
\end{abstract}

